\title{
Entanglement and indistinguishability in a quantum ontology of properties
}

Sebastian Fortin and Olimpia Lombardi

University of Buenos Aires and CONICET

\begin{abstract}
The aim of the present article is to address the problem of entanglement in the case of indistinguishable particles from a perspective based on the algebraic formalism of quantum mechanics, which is the natural formal counterpart of an ontology of properties, devoid of the ontological category of individual. On the basis of this perspective, an algebraic definition of entanglement is adopted, which supplies a unified conception, valid for both the distinguishable and the indistinguishable cases. An additional advantage of this algebraic definition is that it does justice to the relativity of entanglement, a feature that cannot be ignored.
\end{abstract}

\section{1.- Introduction}

Entanglement in many-body systems of so-called "indistinguishable particles" has received a great attention during the last decades due to its applications in quantum information and condensed matter. These works have led to revise the very concept of entanglement in the case of indistinguishability, which poses a specific challenge: according to the traditional definition of entanglement in pure states as non-factorizability, symmetrized and anti-symmetrized states should be entangled (except in some cases involving bosons). But there are good reasons to think that this is not the case: not always non-factorizable states of composite bosonic or fermionic systems should be conceived as legitimate cases of entanglement.

This problem has been addressed from different perspectives, such as modifying the general definition of entanglement, removing the "surplus structure" resulting from the need of symmetrize or anti-symmetrize states in the case of indistinguishability, or treating the entanglement of indistinguishable particles in a different manner than in distinguishable particles. Nevertheless, the common feature of those proposals -as well as of the discussions about the 
general problem of how to understand indistinguishability- is the way in which indistinguishability is conceived: "being indistinguishable from" refers to a relation between particles.

The aim of the present article is to address the problem of entanglement in the case of indistinguishable particles from a different perspective, which does not resort to the concept of particle. The proposal will be to face the issue in the light of an ontology of properties, lacking the ontological category of individual, in the line of that introduced in previous works (Lombardi and Castagnino 2008, da Costa et al. 2013, Lombardi and Dieks 2016). The main goal will be to show that this ontological approach offers a more natural solution to the problem, based on conceiving indistinguishability from a new perspective. To meet this goal, the article is organized

as follows. In Section 2, the problem of entanglement in the case of indistinguishability will be introduced following the usual presentations. In Section 3, some relevant answers to the problem will be revisited. In Section 4, the Hilbert space formalism will be contrasted with the algebraic approach, stressing the different ontological commitments suggested by the two formalisms; this will allow us to introduce our proposal of an ontology of properties, which lacks the ontological category of individual. From this ontological perspective, in Section 5 indistinguishability will be reconceptualized by grounding it on the nature of properties, which can naturally be only numerically different; this ontological feature will be transferred to the quantum formalism, leading to a specific version of the Indistinguishable Postulate. On the basis of this proposal, Section 6 will be devoted to revisit the concept of entanglement, stressing its relational nature. This will lead us to advocate for a characterization of entanglement, formulated in the ontologically motivated algebraic formalism, which will make possible to address entanglement of distinguishable and indistinguishable systems on an equal footing.

\section{2.- Entanglement and indistinguishability: stating the problem}

Although the issue of defining entanglement for mixed states is still an open question (see Earman 2015), there is in general some consensus regarding pure states: a state $\left|\psi_{12}\right\rangle$ of a 
composite system is entangled iff it is non-factorizable in terms of the tensor product of the states $\left|\psi_{1}\right\rangle$ and $\left|\psi_{2}\right\rangle$ of the component systems (see, e.g., Myrvold 2018):

$$
\left|\psi_{12}\right\rangle \neq\left|\psi_{1}\right\rangle \otimes\left|\psi_{2}\right\rangle
$$

This characterization of entanglement works fine when the component systems are distinguishable, but in the case of indistinguishability difficulties appear (despite the generic case introduces some additional technical problems, here we will be confined to $N=2$ because it is sufficient for our conceptual discussion).

First let us clarify that the term 'indistinguishable particles' will be used to denote particles of the same kind, that is, to refer to what in physics is commonly called 'identical particles', that is, particles with the same state-independent properties. Let us consider a composite system $S$, whose component systems are $S_{1}$ and $S_{2}$, corresponding to the Hilbert spaces $\mathcal{H}_{1}$ and $\mathcal{H}_{2}$, respectively. As it is well known, if $S_{1}$ and $S_{2}$ are distinguishable, the Hilbert space of $S$ is $\mathcal{H}=\mathcal{H}_{1} \otimes \mathcal{H}_{2}$. However, if $S_{1}$ and $S_{2}$ are indistinguishable particles, according to the Symmetrization Postulate, the Hilbert space of $S$ is the symmetric subspace $\mathcal{H}_{B}$ of $\mathcal{H}$ in the case of bosons, and the anti-symmetric subspace $\mathcal{H}_{F}$ of $\mathcal{H}$ in the case of fermions. The vectors belonging to $\mathcal{H}_{B}$ and $\mathcal{H}_{F}$ are eigenvectors of the permutation operator $P$, which is defined as follows: given a basis $\left\{\left|\alpha_{i}\right\rangle_{1} \otimes\left|\beta_{j}\right\rangle_{2} \equiv\left|\alpha_{i}\right\rangle_{1}\left|\beta_{j}\right\rangle_{2}\right\}$ of $\mathcal{H}=\mathcal{H}_{1} \otimes \mathcal{H}_{2}$,

$$
P\left(\left|\alpha_{i}\right\rangle_{1}\left|\beta_{j}\right\rangle_{2}\right)=\left|\beta_{j}\right\rangle_{1}\left|\alpha_{i}\right\rangle_{2}
$$

with $\left|\alpha_{i}\right\rangle_{1},\left|\beta_{j}\right\rangle_{1} \in \mathcal{H}_{1}$ and $\left|\beta_{j}\right\rangle_{2},\left|\alpha_{i}\right\rangle_{2} \in \mathcal{H}_{2}$. This operator satisfies the following properties: $P^{2}=I$ and, therefore, $P=P^{-1} ; P$ is unitary, that is, $P P^{\dagger}=P^{\dagger} P=I$ and, therefore, $P$ is Hermitian, $P=P^{\dagger}$. Since $P^{2}=I$, its eigenvalues are +1 and -1 , with symmetric eigenvectors $\left|\mathcal{S}_{i}\right\rangle$ and anti-symmetric eigenvectors $\left|\mathcal{A}_{i}\right\rangle$, respectively, such that

$$
\begin{aligned}
& P\left|\mathcal{S}_{i}\right\rangle=\left|\mathcal{S}_{i}\right\rangle, \quad \text { with }\left|\mathcal{S}_{i}\right\rangle \in \mathcal{H}_{B}, \\
& P\left|\mathcal{A}_{i}\right\rangle=-\left|\mathcal{A}_{i}\right\rangle, \quad \text { with }\left|\mathcal{A}_{i}\right\rangle \in \mathcal{H}_{F} .
\end{aligned}
$$


The projection of any vector $|\Psi\rangle \in \mathcal{H}=\mathcal{H}_{1} \otimes \mathcal{H}_{2}$ onto the subspaces $\mathcal{H}_{B}$ and $\mathcal{H}_{F}$ is performed by the symmetrizer projector $S$ and the anti-symmetrizer projector $A$, respectively, such that ${ }^{1}$

$$
\begin{aligned}
& S|\Psi\rangle=\left|\mathcal{S}_{\Psi}\right\rangle \quad \text { with } S\left|\mathcal{S}_{\Psi}\right\rangle=\left|\mathcal{S}_{\Psi}\right\rangle, \\
& A|\Psi\rangle=\left|\mathcal{A}_{\Psi}\right\rangle \quad \text { with } A\left|\mathcal{A}_{\Psi}\right\rangle=\left|\mathcal{A}_{\Psi}\right\rangle .
\end{aligned}
$$

They satisfy the following properties: they are projectors, $S^{2}=S$ and $A^{2}=A$; they are Hermitian, $S=S^{\dagger}$ and $A=A^{\dagger}$; and $S A=A S=0$. They are related to the permutation operator as

$$
\begin{aligned}
& S=1 / 2(I+P), \\
& A=1 / 2(I-P)
\end{aligned}
$$

Therefore, since in this case $N=2$, then $S+A=I$, which implies that $\mathcal{H}=\mathcal{H}_{B} \oplus \mathcal{H}_{F}$.

Now let us consider the possible states $|\uparrow\rangle_{1},|\downarrow\rangle_{1},|\uparrow\rangle_{2},|\downarrow\rangle_{2}$ of two particles (the labels refer to the Hilbert spaces to which the states belong). If the particles are distinguishable, the definition of entanglement in terms of factorizability works fine: the state of the composite system is entangled iff it is non-factorizable, e.g. (the symbol $\otimes$ is omitted),

$$
\left|\Theta_{\text {ent }}\right\rangle=1 / \sqrt{2}\left(|\uparrow\rangle_{1}|\downarrow\rangle_{2}-|\downarrow\rangle_{1}|\uparrow\rangle_{2}\right)
$$

and it is non-entangled iff it is factorizable, e.g.,

$$
\left|\Theta_{\text {non-ent }}\right\rangle=|\uparrow\rangle_{1}|\downarrow\rangle_{2}
$$

By contrast, if the two particles are indistinguishable, the situation is not that clear. Let us focus on the case of two fermions. There are non-factorizable states that are uncontroversially accepted as entangled since obtained by the anti-symmetrization of a non-factorizable state; for example,

$$
\begin{gathered}
\left|\Psi_{\text {ent }}\right\rangle=1 / \sqrt{4}\left(|L\rangle_{1}|\uparrow\rangle_{1}|R\rangle_{2}|\downarrow\rangle_{2}-|L\rangle_{1}|\downarrow\rangle_{1}|R\rangle_{2}|\uparrow\rangle_{2}-\right. \\
\left.|R\rangle_{1}|\downarrow\rangle_{1}|L\rangle_{2}|\uparrow\rangle_{2}+|R\rangle_{1}|\uparrow\rangle_{1}|L\rangle_{2}|\downarrow\rangle_{2}\right),
\end{gathered},
$$

\footnotetext{
${ }^{1}$ Let us remind that the symmetrizer and the anti-symmetrizer operators must normalize the state or, otherwise, the state must be normalized a posteriori (see, e.g., Messiah 1961).
} 
where $|L\rangle$ and $|R\rangle$ represent spatial locations left and right. This is a legitimate anti-symmetric state since $P\left|\Psi_{\text {ent }}\right\rangle=-\left|\Psi_{\text {ent }}\right\rangle$ and it can be obtained from the anti-symmetrization of the nonanti-symmetric non-factorizable state $\left|\psi_{12}\right\rangle$ :

$$
\left|\Psi_{\text {ent }}\right\rangle=A\left|\Psi_{12}\right\rangle=A\left[\frac{1}{\sqrt{2}}\left(|L\rangle_{1}|\uparrow\rangle_{1}|R\rangle_{2}|\downarrow\rangle_{2}-|L\rangle_{1}|\downarrow\rangle_{1}|R\rangle_{2}|\uparrow\rangle_{2}\right)\right]
$$

However, factorizable states of the form of eq. (10) are not legitimate in the case of indistinguishable particles. In the case of two fermions, a factorizable vector as $\left|\Psi_{12}\right\rangle=|L\rangle_{1}|\uparrow\rangle_{1}|R\rangle_{2}|\downarrow\rangle_{2}$ must be anti-symmetrized,

$$
\left|\Psi_{\text {tang }}\right\rangle=A\left|\Psi_{12}\right\rangle=1 / \sqrt{2}\left(|L\rangle_{1}|\uparrow\rangle_{1}|R\rangle_{2}|\downarrow\rangle_{2}-|R\rangle_{1}|\downarrow\rangle_{1}|L\rangle_{2}|\uparrow\rangle_{2}\right)
$$

which is a legitimate anti-symmetric state since $P\left|\Psi_{\text {tang }}\right\rangle=-\left|\Psi_{\text {tang }}\right\rangle$. Muller and Leegwater (2020) call this kind of states, whose non-factorizability is the result of symmetrization or antisymmetrization, tangled. The question is, then, whether tangled states are entangled or not.

\section{3.- Reactions to the problem}

The simplest answer to the problem is to preserve the definition of entanglement as nonfactorizability even in the indistinguishable case (see, e.g. Lévi-Leblond quoted in Ghirardi 2005: 340). However, as Ladyman, Linnebo, and Bigaj (2013) clearly explain, there are good reasons to reject this view: tangled states do not give rise to correlations that violate Bell's inequalities, the probability distribution associated with them factorizes, they are fully compatible with local hidden-variable models, and they cannot be used as resources for quantum information processing as legitimate entangled states.

In a detailed and repeatedly referenced paper on entanglement of composite quantum systems, Ghirardi, Marinatto, and Weber (2002) (see also Ghirardi and Marinatto 2003, 2004, 2005) explicitly criticize the definition of entanglement as non-factorizability, and offer a new characterization that intends to be valid for both the distinguishable and the indistinguishable case. Inspired by the idea of "element of physical reality" as proposed in the EPR paper (Einstein et al 1935), the authors state that two systems are non-entangled when they possess a complete 
set of properties. In the distinguishable case, this definition is equivalent to the factorizability of the state. In the indistinguishable case, although factorizability cannot be the criterion of nonentanglement due to symmetrization, this fact "does not forbid to attribute a complete set of physical properties to the subsystems: the only claim that one cannot make is to attribute the possessed property to one rather than to the other constituent." (Ghirardi and Marinatto 2003: 383). In other words, even if one cannot say that "particle 1 has a complete set of properties and particle 2 also has a complete set of properties", one can meaningfully say that "there are two particles, each one with its complete set of properties".

In his analysis of Ghirardi, Marinatto, and Weber's proposal, Friebe (2014) shows that, although proposing a generic criterion for non-entanglement -possessing a complete set of properties-, the authors appeal to a kind of double standard: they use labels in different ways in the distinguishable and the indistinguishable cases. According to them, two distinguishable particles, say an electron and a proton, are individuated by their different state-independent properties. So their individuality is independent of the state: they can share their state-dependent properties. But the authors cannot use that individuation criterion for indistinguishable particles, since they share all their state-independent properties -and, in the case of bosons, they can share all their properties-. Friebe points out that this difference in the individuation criterion leads to a difference in the use of labels: "The talk of «distinguishing» particles by telling «which is which» is ambiguous, because GMW use two different sorts of labels, namely labels according to the description theory of proper names, in the case of non-similar particles, and labels according to the direct reference theory of proper names in the case of similar particles.” (Friebe 2014: 93). In fact, in the case of two distinguishable particles, labels pick out their referents via a description of their state-independent properties: for example, an electron and a proton receive different labels in virtue of their different mass and charge. By contrast, in the case of indistinguishability, two particles cannot be endowed with different names via their different complete sets of properties. Therefore, "when one uniquely picks out a particular particle, to be conventionally called «1» or «2», one cannot say with which set of state-dependent properties it is coupled." (Friebe 2014: 94). In order to recover consistence, Friebe adopts the "summing defense" of Leibniz's principle, according to which there are no indistinguishable particles in an entangled state: the interaction 
unifies the numerically distinct individuals into an undivided whole. This view points to the same direction as our proposal (which will be developed in Section 5) although we ground it on an ontology without individuals.

On the basis of their definition of entanglement, Ghirardi and collaborators prove that what we called tangled states are always non-entangled (see Theorems 2.1 and 2.2 in Ghirardi and Marinatto 2004: 2-3). They also show that these states cannot violate Bell's inequalities (see Ghirardi and Marinatto 2004: 3). However, in a kind of appendix of their recent paper, Muller and Leegwater (2020) argue that this conclusion is, at least, controversial. They consider a product state of the form

$$
\left|\Xi_{\text {prod }}\right\rangle=1 / \sqrt{2}\left(|L\rangle_{1}|\uparrow\rangle_{1}+|R\rangle_{1}|\uparrow\rangle_{1}\right) 1 / \sqrt{2}\left(|L\rangle_{2}|\downarrow\rangle_{2}+|R\rangle_{2}|\downarrow\rangle_{2}\right) \text {. }
$$

Anti-symmetrization turns this state into

$$
\begin{aligned}
\left|\Xi_{\text {tang }}\right\rangle=1 / \sqrt{2} & \left(|L\rangle_{1}|L\rangle_{2}+|R\rangle_{1}|R\rangle_{2}+|L\rangle_{1}|R\rangle_{2}+\right. \\
& \left.+|R\rangle_{1}|L\rangle_{2}\right) 1 / \sqrt{2}\left(|\uparrow\rangle_{1}|\downarrow\rangle_{2}-|\downarrow\rangle_{1}|\uparrow\rangle_{2}\right)^{.}
\end{aligned}
$$

On the basis of Ghirardi and collaborators' criterion for non-entanglement, state $\left|\Xi_{\text {tang }}\right\rangle$, since tangled, should be non-entangled and, as a consequence, should not violate Bell's inequalities. But, according to the authors, $\left|\Xi_{\text {tang }}\right\rangle$ does violate Bell's inequalities. So, they close their article with the question: "Were Ghirardi et al. mistaken to propound that, contra communis opinio, tangled states do not deserve to be called 'entangled' because they do not lead to a violation of some Bell inequality?" (Muller and Leegwater 2020: 15).

In order to answer that question it is necessary to verify the correlations between the values of spin in two different spatial locations $L$ and $R$. So, the state's components containing $|L\rangle_{1}|L\rangle_{2}$ and $|R\rangle_{1}|R\rangle_{2}$ should not be taken into consideration and only the components containing $|L\rangle_{1}|R\rangle_{2}$ and $|R\rangle_{1}|L\rangle_{2}$ should be retained. For this purpose, a previous measurement should be performed, which discriminates between particles detected in the same location and particles detected in different locations. And this previous measurement amounts to collapsing the state of eq. (15) onto the state of eq. (11), which is uncontroversially considered entangled and violates 
Bell's inequalities. But does it mean that the original state of eq. (15) is entangled? The answer is not as clear as Muller and Leegwater seem to suppose.

When facing the general problem of entanglement in (anti-)symmetrized states, some authors consider that the very concept of entanglement in a system of indistinguishable particles is fundamentally different from that in a system of distinguishable particles (see, e.g., Shi 2003, Amico et al. 2008). By contrast, Ladyman, Linnebo, and Bigaj (2013) conceive the problem as a consequence of a formalism possessing too much structure. They claim that the apparent entanglement of tangled states has no physical significance, since it is just an artifact of what Michael Redhead (1980) called "surplus structure" of a formalism, in this case the Hilbert-space formalism. On the basis of this idea, instead of using a tensor product $\mathcal{H} \otimes \mathcal{H}$ subject to the constraint of anti-symmetry to represent a two-fermion system, the authors appeal to the Grossman or exterior algebra $\mathcal{H} \wedge \mathcal{H}$, whose objects are equivalence classes of vectors belonging to $\mathcal{H} \otimes \mathcal{H}$. The equivalent classes are defined in terms of a relation $\sim$ on $\mathcal{H} \otimes \mathcal{H}$

$$
u \otimes v \sim-v \otimes u
$$

In other words, $\mathcal{H} \wedge \mathcal{H}$ is the quotient space $\mathcal{H} \otimes \mathcal{H} / \sim$. The object $u \wedge v \in \mathcal{H} \wedge \mathcal{H}$ is the equivalence class of $u \otimes v \in \mathcal{H} \otimes \mathcal{H}$. Therefore, the projection $\Pi: \mathcal{H} \otimes \mathcal{H} \rightarrow \mathcal{H} \wedge \mathcal{H}$ eliminates many dimensions from $\mathcal{H} \otimes \mathcal{H}$-the surplus structure. The wedge product is, then, defined as ${ }^{2}$

$$
u \wedge v=u \otimes v-v \otimes u,
$$

with specific properties: alternatingness, anticommutativity, homogeneity, and distributivity. Let us apply this formalism to entangled and tangled sates of fermions. The entangled state $\left|\Psi_{\text {ent }}\right\rangle$ of eq. (11) is mapped onto

$$
\begin{array}{r}
\Pi\left|\Psi_{\text {ent }}\right\rangle=1 / 2\left(|L\rangle_{1}|\uparrow\rangle_{1} \wedge|R\rangle_{2}|\downarrow\rangle_{2}-|L\rangle_{1}|\downarrow\rangle_{1} \wedge|R\rangle_{2}|\uparrow\rangle_{2}-\right. \\
\left.|R\rangle_{1}|\downarrow\rangle_{1} \wedge|L\rangle_{2}|\uparrow\rangle_{2}+|R\rangle_{1}|\uparrow\rangle_{1} \wedge|L\rangle_{2}|\downarrow\rangle_{2}\right)
\end{array} .
$$

The application of anticommutativity leads to

$$
\Pi\left|\Psi_{\text {ent }}\right\rangle=\left(|L\rangle_{1}|\uparrow\rangle_{1} \wedge|R\rangle_{2}|\downarrow\rangle_{2}-|L\rangle_{1}|\downarrow\rangle_{1} \wedge|R\rangle_{2}|\uparrow\rangle_{2}\right),
$$

\footnotetext{
${ }^{2}$ States must be normalized also in this case.
} 
which cannot be factorized as a wedge product of two states. By contrast, the tangled state $\left|\Psi_{\text {tang }}\right\rangle$ of eq. (13) is mapped onto

$$
\Pi\left|\Psi_{\text {tang }}\right\rangle=1 / 2\left(|L\rangle_{1}|\uparrow\rangle_{1} \wedge|R\rangle_{2}|\downarrow\rangle_{2}-|R\rangle_{1}|\downarrow\rangle_{1} \wedge|L\rangle_{2}|\uparrow\rangle_{2}\right) .
$$

The application of the anticommutativity leads to

$$
\Pi\left|\Psi_{\text {tang }}\right\rangle=|L\rangle_{1}|\uparrow\rangle_{1} \wedge|R\rangle_{2}|\downarrow\rangle_{2}
$$

which is the wedge product of two states. In this way, Ladyman, Linnebo, and Bigaj (2013) can retain non-factorizability, now in terms of the wedge product, as a criterion to identify entangled states of indistinguishable particles, making it to agree with the criterion based on violation of Bell's inequalities.

Let us apply the wedge product strategy to the tangled state $\left|\Xi_{\text {tang }}\right\rangle$ of eq. (15), which, according to Muller and Leegwater (2020), should be considered entangled against Ghirardi and collaborators' criterion. As the result, $\left|\Xi_{\text {tang }}\right\rangle$ can be expressed in a wedge factorizable form:

$$
\left|\Xi_{\text {tang }}\right\rangle=\left(|L\rangle_{1}|\uparrow\rangle_{1}+|R\rangle_{1}|\uparrow\rangle_{1}\right) \wedge\left(|L\rangle_{2}|\downarrow\rangle_{2}+|R\rangle_{2}|\downarrow\rangle_{2}\right) \text {. }
$$

In other words, if the criterion of entanglement is non-factorizability in terms of the wedge product, the state tangled $\left|\Xi_{\text {tang }}\right\rangle$ of eq. (15) is non-entangled, in agreement with Ghirardi and collaborators' criterion.

A common feature of all these positions is that, despite the differences, the problem is always posed in terms of particles and their states: indistinguishability is conceived as a relation between particles, and it is accepted that the states of systems that are composed of indistinguishable particles must be symmetrized or anti-symmetrized. The question is whether the problem can be approached from a different conception of indistinguishability, which leads to a different view of entanglement applicable in the same way both to the distinguishable and to the indistinguishable cases. 


\section{4.- Formalism and ontology}

A moral that can be drawn from the paper by Ladyman, Linnebo, and Bigaj (2013) is that the formalism of a theory is not neutral when foundational matters are discussed. Therefore, taking a different formal perspective can change the nature of the discussion.

According the traditional story about indistinguishability, since quantum particles of the same kind obey Bose-Einstein or Fermi-Dirac statistics; then, under the assumption of equiprobability, they must be conceived as indistinguishable. As a consequence, all quantum states differing only by a permutation of indistinguishable particles must be observationally indistinguishable. This idea is expressed by the so-called Indistinguishable Principle (IP), whic can be expressed as (for this formulation, see Butterfield 1993):

IP: If the vector $|\psi\rangle$ represents the state of a composite system whose components are indistinguishable particles, then the expectation value of any observable represented by an operator $O$ must be the same for $|\psi\rangle$ and for any permutation $\left|\psi^{\prime}\right\rangle=P|\psi\rangle:\langle O\rangle_{|\psi\rangle}=\langle O\rangle_{\left|\psi^{\prime}\right\rangle}$.

Two well-known ways to satisfy PI is restricting states to be symmetric (bosons) or antisymmetric (fermions), which obey Bose-Einstein or Fermi-Dirac statistics, respectively; so, the Symmetrization Postulate (SP) is introduced.

Although this is, approximately, the traditional story, a closer inspection brings to the light different views. Some think that IP rules out both non-symmetric states and non-symmetric observables (Readhead and Teller 1992), whereas others maintain that it poses a restriction only on observables (Messiah and Greenberg 1964, Huggett and Imbo 2009). It has also been claimed that IP is not a postulate because it is the definition of indistinguishability (Belousek 2000), or because it follows from SP (Muller and Leegwater 2020). In turn, some consider that SP and IP are independent because SP applies to states and IP applies to observables (Belousek 2000), and they search for a justification of SP independent of IP (Bigaj 2020). Furthermore, this debate is seasoned with the discussions about whether the ruled-out vectors/operators only represent empirically inaccessible states/observables or do not represent states/properties at all. 
The point we want to stress here is that these discussions are almost always framed in the Hilbert-space formalism, according to which a quantum system is represented by a Hilbert space. From a formal viewpoint, the Hilbert space is the basic element of the theory: states, represented by vectors, are logically prior; observables are logically posterior since represented by operators acting on those previously defined vectors. In general, this logical priority of states over observables is implicitly endowed with an ontological content: quantum systems belong to the ontological category of individual, and observables belong to the ontological category of property; and given the metaphysical priority of individuals over properties, the ontological priority of systems with their states over observables turns out to be "natural". Of course, this "naturalness" begins to totter as soon as indistinguishability enters the scene, because quantum systems seem to lack a principle on individuality that identify them as a particular individual different from another and as the same particular individual over time. This is what triggers the huge amount of literature about individuals, non-individuals, quasi-individuals, identity, nonidentity, labels, strong distinguishability, weak indistinguishability, Leibniz's Principle of the Identity of Indiscernibles, and many others distinctions directed to cope with the nature of "indistinguishable" particles (see, e.g. French 2019 and the references therein).

Even without undermining the value of traditional discussions, maybe it is time to approach the problem from a different formal perspective: the algebraic formalism of quantum physics does not a priori make use of Hilbert spaces (see, e.g., Haag 1993). According to the algebraic formalism, a quantum system is represented by a *-algebra $\mathcal{O}$ of observables. Since observables are not defined by their action on a Hilbert space, an abstract notion for the adjoint of an operator is required, which is provided by an involution operation $O \rightarrow O^{*}$, with $O \in \mathcal{O}$. The algebra is assumed to be closed under products, linear combinations, and the involution. Moreover, it contains an identity $I \in \mathcal{O}$ that satisfies $I^{*}=I$ and $I O=O$ for all $O \in \mathcal{O}$. A state of the system represented by $\mathcal{O}$ is an expectation-value functional $\omega: \mathcal{O} \rightarrow \mathbb{C}$ belonging to the dual space $\mathcal{O}^{\prime}$ : it is normed, $\omega(I)=1$, and positive, $\omega(O) \geq 0$, for all $O \in \mathcal{O}$. A state $\omega$ is said to be pure if it cannot be written as a non-trivial convex combination $\omega=\lambda_{1} \omega_{1}+\lambda_{2} \omega_{2}$, with $0<\lambda_{1}, \lambda_{2}<1$, $\lambda_{1}+\lambda_{2}=1$, and $\omega_{1}, \omega_{2} \in \mathcal{O}^{\prime}$; otherwise $\omega$ is said to be mixed. 
In the algebraic formalism observables are objects that are logically prior to states. In the particular case that $\mathcal{O}$ is a $\mathrm{C}^{*}$-algebra, the Gelfand-Naimark-Segal (GNS) theorem (Gelfand and Naimark 1943, Segal 1947) proves that $\mathcal{O}$ has a representation in a Hilbert space $\mathcal{H}$ : states are represented by normed trace (density) operators $\rho$ on $\mathcal{H}$, and observables are represented by Hermitian operators $O$ on $\mathcal{H}$. When the state $\omega$ represented by the density operator $\rho$ is pure, there is a vector $|\Psi\rangle \in \mathcal{H}$ such that $\rho=|\Psi\rangle\langle\Psi|$. For simplicity, here we will remain in the context of the $\mathrm{C}^{*}$-algebra case and its Hilbert space representation; in this way our argumentation will stand close to the usual terminology in the discussions about indistinguishability. Nevertheless, it must be stressed that the algebraic formalism is not confined to the Hilbert space

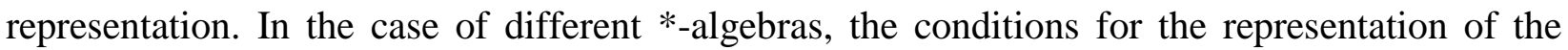
algebra have to be established; for instance, by means of a generalization of the GNS theorem it can be proved that a nuclear algebra can be represented by a rigged Hilbert space (Iguri and Castagnino 1999; for applications of rigged Hilbert spaces to quantum mechanics, see Bohm and Gadella 1989).

In what follows, the abstract algebraic language will not be distinguished from the language of the representation; so, it will be said that a quantum system is given by the algebra $\mathcal{O}$ of observables $O \in \mathcal{O}$, and the system's states are the expectation-value functionals $\rho(O)=\operatorname{Tr}(O \rho)=\langle O\rangle_{\rho} \in \mathbb{R}$, for all $O \in \mathcal{O}$.

Independently of more technical matters, the algebraic approach emphasizes the difference between observables and states, ${ }^{\mathbf{3}}$ whose corresponding spaces may not be the same; this is precisely the case in nuclear algebras, represented by rigged Hilbert spaces. In this theoretical framework, observables are the basic elements of the theory; states are secondary elements, defined in terms of the basic ones. To the extent that states are defined as expectation-value functionals on the algebra of observables, their "nature" is exhausted in fulfilling the task of computing the expectation values of the observables of the algebra. In other words, states must not be confused with observables, they must not be understood as any kind of property of the

\footnotetext{
${ }^{3}$ From now on, we will not distinguish between the physical language (e.g., observables, states) and the mathematical language (e.g., elements of an algebra, functionals on an algebra), under the assumption that the context clarifies the sense of each term.
} 
quantum system. As Earman (2015: 324) stresses, one should never forget "the mantra of the algebraic approach: a system state is an expectation value functional on the system algebra."

If the logical priority of observables over states is transferred to the ontological domain, the algebra of observables turns out to embody the representation of the elemental items of the ontology and the way in which they are arranged in a structure. In fact, on the basis of the algebraic framework, an ontology of properties for quantum mechanics has been proposed in previous papers (Lombardi and Castagnino 2008, da Costa et al. 2013, da Costa and Lombardi 2014, Lombardi and Dieks 2016). More precisely, the ontological counterparts of observables are instances of universal type-properties, each of which has possible case-properties, represented by the eigenvalues of the corresponding observable. ${ }^{4}$ In turn, given an instance of a universal typeproperty, no more than one of its possible case-properties becomes actual. For instance, energy is an example of universal type-property, which can be instantiated as the energy of one system or of another system; in turn, each instantiated type-property has possible case-properties, which constitute the energy spectrum of the system under consideration. From the algebraic perspective, once the quantum system is represented by an algebra of observables, its natural ontological correlate is a bundle of properties, precisely, a bundle of instances of universal type-properties. The quantum state of the system, in turn, is nothing beyond a codification of the probabilities of actualization -interpreted as the measure of the propensities to actualization- for all the possible case-properties of all the instances of universal type-properties belonging to the bundle-system (for a formal presentation of the ontology, see da Costa et al. 2013; for a philosophical elucidation, see Lombardi and Dieks 2016).

The idea of bundle of properties has appeared a few times in the literature on quantum physics. It has been proposed for quantum field theory in its algebraic version by Kuhlmann (2010), and suggested by Friebe (2014) when analyzing the ontological implications of Ghirardi and collaborators' approach. However, in the quantum context it is central to emphasize the difference between the traditional bundle theory and the present bundle proposal. In metahysics, the bundle theory is a "bundle theory of substance" (O'Leary-Hawthorne 1995), or a "bundle

4 The difference between type-properties and case-properties runs parallel to the distinction between determinables and determinates (see, e.g., Wilson 2017). 
theory of individuals" (Dasgupta 2009: 47), or a "bundle view of objects" (French 2019): the aim is to construct individuals (or objects or substances) out of properties; so, some properties that play the role of the principle on individuality, supplying synchronic and diachronic identity, need to be identified. Our bundle view, by contrast, completely dispenses with the ontological category of individual: bundles of properties do not behave as individuals at all since they belong to a different ontological category. Neither do the Leibniz's principle of the identity of Indiscernibles nor the Kantian category of quantity apply to them, since when they combine, they can be neither counted nor reidentified in the composite bundle.

This ontological picture of non-individual bundles of properties cannot be adequately captured by any formal theory which includes, as some of its primitive symbols, individual constants and/or variables referring to objects, whether countable -as in first order logic or Zermelo-Fraenkel set theory- or not -as in quasi-set theory (see, e.g., Krause 1992, da Costa and Krause 1999). An ontology whose elemental items are properties requires a logical formalism in the spirit of the "calculus of relations" proposed by Tarski (1941), where individual variables are absent (see discussion in Lombardi and Dieks 2016).

An ontology of properties that completely bypasses individuals and simply constructs facts out of properties is proposed by Dasgupta (2009) under the name of "generalism"; however, although the author finds inspiration in physics, quantum mechanics does not play any role in his proposal. As noticed by French (2020), this view of non-individual bundles finds a natural resonance with ontic structural realism, which was mainly motivated by the ontological challenges of quantum mechanics (Ladyman 1998). From this perspective, physical objects are not elemental items of the ontology, but rather are "reduced to mere 'nodes' of the structure, or 'intersections' of the relevant relations" (French 2006: 173). French and Ladyman also notice the limitations of traditional languages to describe this kind of ontology, due to the "the descriptive inadequacies of modern logic and set theory which retains the classical framework of individual objects represented by variables and which are the subject of predication or membership respectively" (2003: 41). 
As several authors have stressed, metaphysics is underdetermined by physics due to the fact that quantum mechanics is compatible with different ontological pictures, in particular, one containing individuals and another without them (see, e.g., van Fraassen 1985, 1991; French 1989). In general, the pros and cons of these ontological pictures are discussed in the light of the problem of indistinguishability. By contrast, the present proposal of non-individual bundles aspires to provide a "global" approach: it intends to offer answers to the different ontological challenges of quantum mechanics in terms of a single ontology. Although this does not undermine metaphysical underdetermination, it may offer additional arguments in favor of an ontology of properties without individuals. In fact, conceiving quantum systems as nonindividual bundles of type-properties has the advantage of being immune to the challenge represented by the Kochen-Specker theorem, since this theorem imposes constraints on actual case-properties but introduces no conditions on type-properties. On the other hand, nonseparability naturally follows from the fact that a composite system is a single non-individual bundle, in which the bundle-components cannot be reidentified (for these arguments, see Lombardi 2019). Since here the focus is indistinguishability, the following section will draw the attention towards how this ontological view offers a new approach to this particular challenge.

\section{5.- Indistinguishability in an ontology of properties}

In the algebraic framework, the quantum system is represented by an algebra of observables; then, given two component systems $S_{1}$ and $S_{2}$, represented by the algebras of observables $\mathcal{O}_{1}$ and $\mathcal{O}_{2}$ respectively, the composite system $S$ is represented by $\mathcal{O}_{1} \vee \mathcal{O}_{2}$, the minimal algebra generated by $\mathcal{O}_{1}$ and $\mathcal{O}_{2}$. Since ontologically all systems are non-individual bundles of instances of universal type-properties, not only the component systems $S_{1}$ and $S_{2}$ are bundles, but also the composite system is a bundle. And since bundles are not individuals, there is no principle of individuality that preserves their identity in the composition: the composite system is a new bundle, in which the identity of the components is not retained (see da Costa et al. 2013).

If the two component systems $S_{1}$ and $S_{2}$ are indistinguishable, then $\mathcal{O}_{1}=\mathcal{O}_{2}=\mathcal{O}$; this means that the composite system is now represented by the algebra of observables 
$\mathcal{O}_{1} \vee \mathcal{O}_{2}=\mathcal{O} \vee \mathcal{O}$. This means that the observable $O_{1} \in \mathcal{O}_{1}$ corresponding to a certain physical magnitude (say, energy, following with the same example) is indistinguishable from the observable $O_{2} \in \mathcal{O}_{2}$ corresponding to the same physical magnitude; moreover, $O_{1}$ and $O_{2}$ have the same eigenvalues and eigenvectors. In the algebraic framework, all this can be said in the domain of the observables, without reference to states.

When expressed in this way, indistinguishability seems to be defined in the mathematical realm, losing then its physical content. But this is not so: it can be expressed in clear ontological terms in the context of a realm of properties and their bundles. In an ontology of properties, indistinguishability is primarily a relation that holds between two instances of a same universal type-property when they have the same case-properties. In this case, the two instances of the universal type-property are only numerically different. Nevertheless, this is not the reason why they do not satisfy Leibniz's Principle of Identity of Indiscernibles. The principle is not false, but it simply does not apply in this case: whereas it refers to the identity of indiscernible individuals, in our case indistinguishability is a relation between items belonging to the ontological category of property (see Lombardi and Dieks 2016). From this primary meaning of indistinguishability, its derived meaning when applied to non-individual bundles follows directly: two bundle-systems are indistinguishable when their respective instances of universal type-properties are indistinguishable. Again, Leibniz's principle does not apply to bundle-systems because in this case individual systems are not constructed out of them. In turn, when indistinguishable bundlesystems combine, the composite system is just one whole bundle, in which the original bundles can no longer be identified.

Once the category of individual is expelled from the ontology, the IP finds a natural ontological justification. When indistinguishable bundles combine, it is natural to expect that the instances of universal type-properties belonging to the composite bundles do not distinguish between those component bundles. More simply, when two indistinguishable bundles merge into a single whole, which component bundle is taken first and which second does not matter at all. Mathematically, this requires that the observables $O_{c}=\sum_{i j} k_{i j}\left(O_{1 i} \otimes O_{2 j}\right) \in \mathcal{O}_{1} \vee \mathcal{O}_{2}$, representing the instances of universal type-properties belonging to the composite system, are such that $O_{1 i} \otimes O_{2 j}=O_{2 i} \otimes O_{1 j}$. In other words, the observables $O_{c} \in \mathcal{O}_{1} \vee \mathcal{O}_{2}=\mathcal{O} \vee \mathcal{O}$ of the composite 
bundle-system must be symmetric with respect to the permutation of the component bundles. In a previous paper (Lombardi and Castagnino 2008) it has been shown that this ontologically motivated symmetry of observables leads to the desired result expressed by the IP when a state $\rho$ is an expectation value functional acting on an algebra of observables $\mathcal{O}$. Mathematically represented by an operator, the state $\rho_{c}$ of the composite bundle-system can always be decomposed as a sum of two operators, one symmetric and other anti-symmetric with respect to the permutation of indices corresponding to the component bundles: $\rho_{c}=\rho_{c}^{S}+\rho_{c}^{A}$. Therefore, since $O_{c}$ is symmetric, its expectation value in the generic state $\rho_{c}$ is computed as

$$
\rho_{c}\left(O_{c}\right)=\left\langle O_{c}\right\rangle_{\rho_{c}}=\operatorname{Tr}\left(O_{c} \rho_{c}\right)=\operatorname{Tr}\left(O_{c} \rho_{c}^{S}+O_{c} \rho_{c}^{A}\right)=\operatorname{Tr}\left(O_{c} \rho_{c}^{S}\right)=\left\langle O_{c}\right\rangle_{\rho_{c}^{s}}=\rho_{c}^{S}\left(O_{c}\right) .
$$

This means that the non-symmetric part of the state has no effect in its application onto symmetric observables and, as a consequence, it is superfluous. In turn, since any permutation $\rho_{c}^{\prime}=P \rho_{c} P^{\dagger}$ on the state $\rho_{c}$ only modifies its non-symmetric part, then IP follows: $\left\langle O_{c}\right\rangle_{\rho_{c}}=\left\langle O_{c}\right\rangle_{\rho_{c}^{\prime}}$. Let us analyze this argument in more detail.

As introduced in the previous section, the IP imposes the restriction

$$
\mathrm{IP}_{s t}:\langle O\rangle_{|\psi\rangle}=\langle O\rangle_{\left|\psi^{\prime}\right\rangle},
$$

where $\left|\psi^{\prime}\right\rangle=P|\psi\rangle$. However, since the permutation operator $P$ acts on a generic observable $O$ as $O^{\prime}=P^{\dagger} O P$, it is easy to see that

$$
\langle O\rangle_{\left|\Psi^{\prime}\right\rangle}=\left\langle\Psi^{\prime}|O| \Psi^{\prime}\right\rangle=\left\langle\Psi\left|P^{\dagger} O P\right| \Psi\right\rangle=\left\langle\Psi\left|O^{\prime}\right| \Psi\right\rangle=\left\langle O^{\prime}\right\rangle_{|\psi\rangle} .
$$

Then, the IP restriction can be expressed in a mathematically equivalent form:

$$
\mathrm{IP}_{o b s}:\langle O\rangle_{|\psi\rangle}=\left\langle O^{\prime}\right\rangle_{|\psi\rangle}
$$

However, as van Fraassen (1991) emphasizes, these two formulations are not equivalent from an interpretive viewpoint. $\mathrm{IP}_{s t}$ establishes a restriction on the states of the composite system, allowing just symmetric-bosonic states $|\mathcal{S}\rangle$ or anti-symmetric-fermionic states $|\mathcal{A}\rangle$. By contrast, $\mathrm{IP}_{o b s}$ imposes a restriction on the observables of the composite system, allowing just symmetric observables (see Messiah and Greenberg 1964). From the algebraic-based viewpoint of an ontology of properties, $\mathrm{IP}_{o b s}$ is the formulation of the IP that makes sense, since it requires that all 
the observables of a bundle-system, which is composite of indistinguishable bundle-systems, are symmetric with respect to the permutation of the component bundles. Furthermore, from this perspective, $\mathrm{IP}_{o b s}$ is not an ad hoc postulate of the theory but a consequence of the ontologically motivated symmetry of the composite system's observables.

Let us recall that an observable $O_{s y m}$ is symmetric when $O_{s y m}^{\prime}=O_{s y m}$, that is

$$
O_{\text {sym }}^{\prime}=P^{\dagger} O_{\text {sym }} P=O_{\text {sym }} \Leftrightarrow O_{\text {sym }} P=P O_{\text {sym }} \Leftrightarrow\left[P, O_{\text {sym }}\right]=0 .
$$

In turn, since $P$ and $O_{\text {sym }}$ commute, they have a common basis of eigenstates: This means that, since symmetric states $|\mathcal{S}\rangle$ and anti-symmetric states $|\mathcal{A}\rangle$ are eigenstates of $P$ (see eqs. (3) and (4)), they are also eigenstates of $O_{\text {sym }}$ :

$$
O_{\text {sym }}|\mathcal{S}\rangle=s|\mathcal{S}\rangle \quad O_{\text {sym }}|\mathcal{A}\rangle=a|\mathcal{A}\rangle
$$

In other words, symmetric observables preserve the symmetry and anti-symmetry of states. This feature shows an additional advantage of IP $_{o b s}$ over IP $_{s t}$. Given that bosons cannot be transformed into fermions and vice versa, no transition must occur between bosonic symmetric states and fermionic anti-symmetric states during the time-evolution of the composite system. Then, the permutation operator must be invariant under the evolution: $U^{\dagger}(t) P U(t)=P \Leftrightarrow[P, U(t)]=0$. Since the evolution operator is $U(t)=e^{-i H t / \hbar}$, the commutation between $P$ and $U(t)$ implies that $[P, H]=0$ and, as a consequence, that $H$ is a symmetric observable. Therefore, the preservation of the states' symmetry type requires the symmetry of $H$. If the IP is interpreted as $\mathrm{IP}_{s t}$, that is, as a restriction on states, the symmetry of the Hamiltonian must be posited as an additional condition. By contrast, since the Hamiltonian of the composite system is one of its observables, its symmetry follows directly from $\mathrm{IP}_{o b s}$ with no need of a further requirement.

If the symmetric/anti-symmetric state $|\mathcal{S}\rangle /|\mathcal{A}\rangle$ is obtained by symmetrizing/antisymmetrizing a generic state $|\Psi\rangle,|\mathcal{S}\rangle=S|\Psi\rangle /|\mathcal{A}\rangle=A|\Psi\rangle$, respectively, then the expectation value of a generic observable $O$ can be computed as ${ }^{5}$

\footnotetext{
${ }^{5}$ Normalization is also necessary here. See footnote 1.
} 


$$
\begin{aligned}
& \langle O\rangle_{|\mathcal{S}\rangle}=\langle\mathcal{S}|O| \mathcal{S}\rangle=\left\langle\Psi\left|S^{\dagger} O S\right| \Psi\right\rangle=\left\langle\Psi\left|O_{\mathcal{S}}\right| \Psi\right\rangle=\left\langle O_{\mathcal{S}}\right\rangle_{|\psi\rangle}, \\
& \langle O\rangle_{|\mathcal{A}\rangle}=\langle\mathcal{A}|O| \mathcal{A}\rangle=\left\langle\Psi\left|A^{\dagger} O A\right| \Psi\right\rangle=\left\langle\Psi\left|O_{\mathcal{A}}\right| \Psi\right\rangle=\left\langle O_{\mathcal{A}}\right\rangle_{|\Psi\rangle},
\end{aligned}
$$

where $O_{\mathcal{S}} \in \mathcal{O}_{\mathcal{S}} \subset \mathcal{O}_{\text {sym }}$ and $O_{\mathcal{A}} \in \mathcal{O}_{\mathcal{A}} \subset \mathcal{O}_{\text {sym }}$. Moreover, it is easy to prove that the algebras $\mathcal{O}_{\mathcal{S}}$ and $\mathcal{O}_{\mathcal{A}}$ are not only subalgebras but also subspaces of $\mathcal{O}_{\text {sim }}$ : if $O_{\mathcal{S}}, O_{\mathcal{S}}^{\prime} \in \mathcal{O}_{\mathcal{S}}$ and $O_{\mathcal{A}}, O_{\mathcal{A}}^{\prime} \in \mathcal{O}_{\mathcal{A}}$, then $O_{\mathcal{S}}+O_{\mathcal{S}}^{\prime} \in \mathcal{O}_{\mathcal{S}}, O_{\mathcal{A}}+O_{\mathcal{A}}^{\prime} \in \mathcal{O}_{\mathcal{A}}$ and $\lambda O_{\mathcal{S}} \in \mathcal{O}_{\mathcal{S}}, \lambda O_{\mathcal{A}} \in \mathcal{O}_{\mathcal{A}}$. Therefore, the same empirical reason that imposes the restriction to symmetric-bosonic states $|\mathcal{S}\rangle$ or anti-symmetric-fermionic states $|\mathcal{A}\rangle$ in the usual presentations - provided that paraparticles have not been detected-, from the present perspective imposes the restriction to bosonic observables $O_{\mathcal{S}} \in \mathcal{O}_{\mathcal{S}}$ or fermionic observables $O_{\mathcal{A}} \in \mathcal{O}_{\mathcal{A}}$. More precisely, a bosonic/fermionic composite system is a non-individual bundle represented by $\left(\mathcal{O}_{\mathcal{S}}, H_{\mathcal{S}}\right) /\left(\mathcal{O}_{\mathcal{A}}, H_{\mathcal{A}}\right)$, where $H_{\mathcal{S}} \in \mathcal{O}_{\mathcal{S}} / H_{\mathcal{A}} \in \mathcal{O}_{\mathcal{A}}$ is the corresponding bosonic/fermionic Hamiltonian. In summary, when the algebra of observables of a system resulting from the composition of two indistinguishable systems is restricted to be a bosonic algebra or a fermionic algebra, the restriction on states is no longer necessary. Since states are devices designed exclusively to compute expectation values, if two vectors yield the same expectation values for all the observables of the algebra representing the system, they are two mathematical representations of the same physical situation: the two states are physically equivalent.

\section{6.- Entanglement revisited}

Although the phenomenon of entanglement is at the very heart of quantum mechanics, the meaning of the concept still deserves a deep inspection. To begin with, it is necessary to face the question about what the term 'entanglement' is applied to. Commonly it is applied to particles distinguishable or not-, as if being entangled were a relation that links quantum objects to each other. Sometimes, such a relation is conceived as the consequence of an interaction between quantum systems, which persists even when the interaction has ceased. These common views need to be carefully assessed.

First, interaction is not a requirement for entanglement. In fact, in the case of the so-called "entanglement swapping" (first introduced in Żukowski et al. 1993; for its first experimental 
realization, see Pan et al. 1998), entanglement can be generated between systems that have never physically interacted with each other. Second, the idea of entanglement as a relation between particles must be revised since there may be entanglement in a single photon or in a single atom. For example, a kind of "intraparticle entanglement" (Harshman and Wikramasekara 2007: 2) obtains between the momentum degrees of freedom and the spin degrees of freedom of a single atom (see also Harshman 2012) or between two spatial modes of a single photon passing through a beam splitter (Terra Cunha et al. 2007). Furthermore, one-particle entanglement is as good as two-particle entanglement with respect to applications: a one-photon or one-atom state can be used to violate Bell's inequalities and to teleport a qubit (Lee and Kim 2000). On this basis it has been claimed that a "common fallacy is to say that «entanglement is a property of many particle systems». It is, however, correct to say that «entanglement is a property of composite systems», i.e., systems that have more than one subsystem" (Terra Cunha et al. 2007: 2280). In other words, it is not necessary to talk about "particles" when entanglement is considered.

The above remarks point to the fact that entanglement should be conceived not as a relation between particles, but as a property of composite systems, more precisely, of their states. Nevertheless, this does not mean that it is an absolute property of a state. As Earman (2015) clearly argues, a given state is entangled or not only in relation to the decomposition of the composite system into subsystems. In fact, a given state may be entangled with respect to a certain decomposition and non-entangled with respect to another one. The typical case is that of the hydrogen atom, which can be decomposed into the proton-system and the electron-system, but also into the center of mass-system and the relative-system: the entanglement of the atom's state is relative to the chosen decomposition (see, e.g., Dugić and Jeknić-Dugić 2008, Harshman 2012). At present, there is an extensive agreement on that entanglement is a relative notion. As Terra Cunha and collaborators (2007: 2278) claim, "entanglement is a property of a quantum state relative to a given set of subsystems $[\ldots]$ a question like «is there entanglement in such a state?» is meaningless in isolation."; the authors even prove a theorem according to which, given a state vector $|\Psi\rangle$ in a finite-dimensional state space $\mathcal{H}$ with non-prime dimension $d=m n$, there always exists a tensor product structure $\mathcal{H}=\mathcal{H}^{m} \otimes \mathcal{H}^{n}$ with respect to which $|\Psi\rangle$ is factorizable. And since "without further physical assumption, no partition has an ontologically 
superior status with respect to any other" (Zanardi 2001: 4), there are no grounds to privilege a claim about the entanglement of a quantum state over others. By contrast, the discussions about entanglement in the indistinguishable case versus entanglement in the distinguishable case seem to adopt an implicit ontological assumption that prioritizes the partition of the composite system in terms of "particles".

In general, the relativity of entanglement is expressed in terms of different tensor product structures: the different ways in which a Hilbert space can be decomposed into a tensor product of Hilbert spaces. However, several authors have turned their attention from Hilbert spaces to algebras of observables. For instance, Zanardi and collaborators have taken an algebra $\mathcal{M}$ on a finite Hilbert space $\mathcal{H}$ as the starting point, to prove that, given two independent subalgebras $\mathcal{A}$ and $\mathcal{B}$ of $\mathcal{M}$ that satisfy (i) independence $([\mathcal{A}, \mathcal{B}]=0$, that is, $[a, b]=0$ for all $a \in \mathcal{A}$ and $b \in \mathcal{B}$ ) and (ii) completeness $(\mathcal{A} \otimes \mathcal{B} \cong \mathcal{A} \vee \mathcal{B}=\mathcal{M})$, then $\mathcal{A}$ and $\mathcal{B}$ induce a tensor product $\mathcal{H}_{\mathcal{A}} \otimes \mathcal{H}_{\mathcal{B}}$ (Zanardi 2001, Zanardi et al. 2004). The authors stress that, in this way, the partition of the algebra of observables and the resulting entanglement of the state of interest can be made to depend on a particular set of experimental capabilities, that is, on the accessible observables in each situation. In turn, Harshman and Ranade (2011) provide an explicit constructive method for generating those subalgebras from a finite set of operators, which, although may look arbitrary from the viewpoint of the unstructured Hilbert space, have the correct properties to rigorously define locality, separability, and entanglement. This structural perspective on entanglement also dispenses with the concept of particle and places observables in the center of the scene.

The relativity of entanglement adds a new difficulty to the issue of entanglement in the case of indistinguishability. As claimed above, entanglement is a property of a state of a composite system relative to the decomposition of the system into subsystems expressed by a tensor product structure of the Hilbert space. But, in general, the bosonic symmetric Hilbert space $\mathcal{H}_{B}$ and the fermionic anti-symmetric Hilbert space $\mathcal{H}_{F}$ do not admit tensor product structures in terms of the single-particle Hilbert spaces, just as expected from the indistinguishability condition (for the particular cases in which the tensor-product decomposition is possible, see Caulton 2014a). In order to face this problem, among others, Balachandran and his collaborators (2013a, 2013b) offer a completely algebraic approach to entanglement. The guiding idea underlying their 
approach is that, given a composite system $S$ with components $S_{1}$ and $S_{2}$, a pure state $|\Psi\rangle$ of $S$ is non-entangled if and only if the corresponding reduced states of $S_{1}$ and $S_{2}$ are also pure (see Ghirardi et al. 2002). In the context of the Hilbert space formalism, if $S$ is represented by $\mathcal{H}=\mathcal{H}_{1} \otimes \mathcal{H}_{2}$, where $\mathcal{H}_{1}$ and $\mathcal{H}_{2}$ represent the component systems $S_{1}$ and $S_{2}$ respectively, the reduced states $\rho_{1}^{r}$ and $\rho_{2}^{r}$ of $S_{1}$ and $S_{2}$ are computed in terms of the partial traces on $\rho=|\Psi\rangle\langle\Psi|$ over the degrees of freedom of $S_{2}$ and $S_{1}$, respectively. However, it must be recalled that the reduced state $\rho_{1}^{r}$ is defined as the density operator by means of which the expectation values of all the observables belonging to $S_{1}$ can be computed, that is,

$$
\forall O=O_{1} \otimes I_{2} \in \mathcal{O}, \quad\langle O\rangle_{\rho}=\left\langle O_{1}\right\rangle_{\rho_{1}^{r}},
$$

where $\mathcal{O}=\mathcal{H} \otimes \mathcal{H}$ is the algebra of observables of the composite system $S, O_{1} \in \mathcal{O}_{1}=\mathcal{H}_{1} \otimes \mathcal{H}_{1}$ is an observable of $S_{1}, I_{2}$ is the identity in $\mathcal{O}_{2}=\mathcal{H}_{2} \otimes \mathcal{H}_{2}$, and $\rho \in \mathcal{H} \otimes \mathcal{H}$ is the state of $S$. It is on the basis of this definition that the reduced states are computed in terms of partial traces (for a detailed discussion about the conceptual meaning of reduced states, see Fortin and Lombardi 2014). As Schlosshauer (2007: 48) claims in his well-known book about decoherence, this means that, strictly speaking, a reduced density operator is only "a calculational tool" for computing expectation values.

However, when the tensor product structure $\mathcal{H}_{1} \otimes \mathcal{H}_{2}$ cannot be obtained, the operation of partial trace is ill-defined and, as a consequence, reduced states cannot be computed as in the previous case. Neverthless, the definition of the reduced state can be retained. What Balachandran and collaborators show is that the partial trace operation has a natural generalization in the operation of restriction to a subalgebra. Let us consider the algebra of operators $\mathcal{O}$, a state $\omega$ on $\mathcal{O}$, and a partition of subalgebras $\mathcal{O}_{1} \vee \mathcal{O}_{2}$. A state $\omega_{1}^{r}$ is said to be the restriction of $\omega$ to $\mathcal{O}_{1}, \omega_{1}^{r}=\left.\omega\right|_{\mathcal{O}_{1}}: \mathcal{O}_{1} \rightarrow \mathbb{C}$, when

$$
\forall O_{1} \in \mathcal{O}_{1}, \quad\left\langle O_{1} \otimes I_{2}\right\rangle_{\omega}=\left\langle O_{1}\right\rangle_{\omega_{1}^{r}},
$$

where $I_{2}$ is the identity operator of $\mathcal{O}_{1}$. In the authors' terms: "when $\mathcal{H}$ is not of the form of a «simple tensor product,» partial trace is not a suitable operation. In contrast, if the system is described in terms of a state $\omega_{\rho}$ on an algebra $\mathcal{A}$, it is still sensible to describe a subsystem in terms of a corresponding subalgebra $\mathcal{A}_{0}$ and of the restriction $\omega_{\rho, 0}$ of $\omega_{\rho}$ to $\mathcal{A}_{0}$." (Balachandran 
et al. 2013b: 2). Entanglement for pure states can be characterized on this basis: a pure state $\omega$ on $\mathcal{O}$ is non-entangled with respect to the partition $\mathcal{O}_{1} \vee \mathcal{O}_{2}$ if the state restrictions $\omega_{r 1}=\left.\omega\right|_{\mathcal{O}_{1}}$ and $\omega_{r 2}=\left.\omega\right|_{\mathcal{O}_{2}}$ are also pure. It is easy to see that this characterization of entanglement agrees with Ghirardi's characterization -in terms of reduced states computed as partial traces in the Hilbert space formalism- when the composite system can represented as a tensor product structure. This algebraic approach to entanglement is general enough to be applied not only to bosons and fermions, but also to the case of parastatistics and in any other physical situation in which a relevant tensor product structure is not available: "It allows us to meaningfully treat the entanglement of identical and nonidentical particles on an equal footing, without the need to resort to different criteria according to the case under study.” (Balachandran et al. 2013b: 1). ${ }^{6}$

The possibility of treating entanglement in the distinguishable and the indistinguishable cases on an equal footing may count as an advantage of the algebraic approach to entanglement over other approaches to the problem. For instance, Ghirardi, Marinatto, and Weber (2002) explicitly discriminate between entanglement of distinguishable particles (Part II) and of indistinguishable particles (Part III). In turn, entanglement is conceived as a relation between particles, without taking into account the relativity of entanglement. In the case of the proposal of Ladyman, Linnebo, and Bigaj (2013), the need to eliminate the surplus structure by means of the wedge-product construction is a consequence of the Hilbert space formalism. Beyond these particular advantages, our ontologically motivated algebraic approach diverges from those proposals in how quantum systems are conceived and, as a consequence, what meaning is assigned to the concept of indistinguishability.

The characterization of entanglement in the algebraic framework in terms of restriction of the state to a subalgebra does not undermine the appeal to the violation of Bell's inequalities as a criterion for entanglement. This criterion is usually accepted in the literature on quantum foundations, both implicitly or explicitly: "long-distance entanglement, and experimental tests of

${ }^{6}$ Howard Barnum and collaborators (Barnum et al. 2003, 2004, Viola and Barnum 2010) take the observable viewpoint a step further: they introduce a generalization based on the idea that entanglement is relative to a distinguished subspace of observables rather than to a distinguished subsystem decomposition. 
the Bell inequalities provide convincing evidence that it is a feature of reality" (Myrvold et al. 2020). In the case of indistinguishable particles, when Ladyman, Linnebo, and Bigaj (2013) stress that certain anti-symmetric states are entangled, or when Muller and Leegwater (2020) ask whether a tangled state may be entangled, they use the inequalities' violation as the touchstone to identify a case of entanglement. Also for the case of indistinguishability, Caulton (2014b) proves that, if entanglement is defined as by Ghirardi, Marinatto, and Weber (2002), then a pure state of a composite system of two fermions is entangled if and only if it violates a Bell's inequality. Although the above arguments are developed in the context of the Hilbert space formalism, Bell's inequalities involve correlations between probabilities that can be expressed in terms of expectation values, which are magnitudes clearly defined in the algebraic formalism. Moreover, Bell's inequalities have been expressed in terms of commuting operator subalgebras of the total algebra instead of appealing to tensor factors of the total Hilbert space (see, e.g. Baez 1987; see also Scholz and Werner 2008). As a consequence, the close connection between entanglement and Bell's result does not need to be lost in an ontologically motivated algebraic formalism.

\section{7.- Conclusions}

In this article we have discussed the problem of defining entanglement in the case of systems composed of indistinguishable subsystems. After considering different views of the problem, we have proposed an approach based on the algebraic formalism of quantum mechanics, which is the natural formal counterpart of an ontology of properties, devoid of the ontological category of individual. This ontology leads to a different way of conceiving indistinguishability, which is no longer a relation between particles, but primarily a relation between instantiated properties. One advantage of this perspective is that the symmetrization of states is no longer a requirement, and that the symmetry of the Hamiltonian needs not to be added to the symmetrization of states but follows directly from the Indistinguishable Principle.

On the basis of this ontologically motivated perspective, we have adopted an algebraic definition of entanglement, which supplies a unified conception, valid for both the distinguishable and the indistinguishable cases: whether a state is entangled or not has to do not 
with its mathematical form but with the kind of correlations between probabilities (or expectation values) it generates. An additional advantage of this algebraic definition is that it does justice to the relativity of entanglement, a feature that already cannot be ignored: the decomposition of the whole composite system in terms of what seem to be "particles" is not preferred, but is one of its many different possible decompositions, and entanglement is relative to each one of them.

There is no doubt that our language, and perhaps also our thoughts, are strongly anchored to the ontological model of individual objects as primitives and as bearers of properties and relations. For this reason, giving up the category of individual and embracing an ontology of properties requires a philosophical effort. However, given the many challenges that quantum mechanics imposes on us, modifying our intuitive framework might be the lowest price if the new ontological picture dissolves the debates about what indistinguishability of particles means. But the detailed discussion of this more general issue will be the subject of a future work.

\section{References}

Amico, L., Fazio, R., Osterloh, A., and Derdal, V. (2008). "Entanglement in many-body systems." Reviews of Modern Physics, 80: 517-576.

Baez, J. (1987). "Bell's inequality for C*-algebras." Letters in Mathematical Physics, 13: 135136.

Balachandran, A. P., Govindarajan, T. R., de Queiroz, A. R., and Reyes-Lega, A. F. (2013a). “Algebraic approach to entanglement and entropy." Physical Review A, 88: 022301.

Balachandran, A. P., Govindarajan, T. R., de Queiroz, A. R., and Reyes-Lega, A. F. (2013b). "Entanglement and particle identity: A unifying approach." Physical Review Letters. 110: 080503.

Barnum, H., Knill, E., Ortiz, G., Somma, R., and Viola, L. (2004). “A subsystem-independent generalization of entanglement." Physical Review Letters, 92: 107902.

Barnum, H., Knill, E., Ortiz, G., and Viola, L. (2003). “Generalizations of entanglement based on coherent states and convex sets.” Physical Review A, 68: 032308. 
Belousek, D. W. (2000). "Statistics, symmetry, and the conventionality of indistinguishability in quantum mechanics." Foundations of Physics, 30: 1-34.

Bigaj, T. (2020). "How to justify the symmetrization postulate in quantum mechanics." Journal for General Philosophy of Science, on line first https://link.springer.com/article/10.1007/s10838-020-09509-3.

Bohm, A. and Gadella, M. (1989). Dirac Kets, Gamow Vectors and Gel'fand Triplets. The Rigged Hilbert Space Formulation of Quantum Mechanics, Springer Lecture Notes in Physics, vol. 348. Berlin: Springer.

Bokulich, A. and Jaeger, G. (eds.) (2010). Philosophy of Quantum Information and Entanglement. Cambridge: Cambridge University Press.

Butterfield, J. (1993). "Interpretation and identity in quantum theory." Studies in History and Philosophy of Science, 24: 443-476.

Caulton, A. (2014a). "Qualitative individuation in permutation-invariant quantum mechanics." arXiv: 1409.0247.

Caulton, A. (2014b). "Physical entanglement in permutation-invariant quantum mechanics." $\operatorname{arXiv:1409.0246.}$

Cohen-Tannoudji, C., Diu, B., and Laloë, F. (1978). Quantum mechanics (Vol. 2). Hoboken: Wiley.

da Costa, N. and Krause, D. (1999). "Set-theoretical models for quantum systems.” Pp. 171-181 in M. L. dalla Chiara, R. Giuntini and F. Laudisa (eds.), Language, Quantum, Music. Dordrecht: Kluwer.

da Costa, N. and Lombardi, O. (2014). "Quantum mechanics: ontology without individuals." Foundations of Physics, 44: 1246-1257.

da Costa, N., Lombardi, O., and Lastiri, M. (2013). "A modal ontology of properties for quantum mechanics.” Synthese, 190: 3671-3693. 
Dasgupta, S. (2009). "Individuals: an essay in revisionary metaphysics." Philosophical Studies, 145: 35-67.

Dugić, M. and Jeknić-Dugić, J. (2008). "What is «system»: The information-theoretic arguments." International Journal of Theoretical Physics, 47: 805-813.

Earman, J. (2015). "Some puzzles and unresolved issues about quantum entanglement." Erkenntnis, 80: 303-337.

Einstein, A., Podolsky, B., and Rosen, N. (1935). "Can quantum-mechanical description of physical reality be considered complete?" Physical Review, 47: 777-780.

Fortin, S. and Lombardi, O. (2014). "Partial traces in decoherence and in interpretation: What do reduced states refer to?" Foundations of Physics, 44: 426-446.

French, S. (1989). "Identity and individuality in classical and quantum physics." Australasian Journal of Philosophy, 67: 432-446.

French, S. (2006). "Structure as a weapon of the realist." Proceedings of the Aristotelian Society, 106: $167-185$.

French, S. (2019). "Identity and individuality in quantum theory." In E. N. Zalta (ed.), The Stanford Encyclopedia of Philosophy (Winter 2019 Edition), URL = https://plato.stanford.edu/archives/win2019/entries/qt-idind/.

French, S. (2020). "What is this thing called structure? (Rummaging in the toolbox of metaphysics for an answer)." http://philsci-archive.pitt.edu/id/eprint/16921.

French, S. and Ladyman, J. (2003). "Remodelling structural realism: quantum physics and the metaphysics of structure." Synthese, 136: 31-56.

Friebe, C. (2014). "Individuality, distinguishability, and (non-)entanglement: A defense of Leibniz's principle." Studies in History and Philosophy of Modern Physics, 48: 89-98.

Gelfand, I. and Naimark, M. (1943). "On the imbedding of normed rings into the ring of operators in Hilbert space.”. Matematicheskii Sbornik, 54: 197-217. 
Ghirardi, GC. (2005). Sneaking a Look at God's Cards: Unraveling the Mysteries of Quantum Mechanics. Princeton NJ: Princeton University Press.

Ghirardi, GC. and Marinatto, L. (2003). "Entanglement and properties." Fortschritteder Physik, 51: 379-387.

Ghirardi, GC. and Marinatto, L. (2004). "General criterion for the entanglement of two indistinguishable particles." Physical Review A, 70: 12109.

Ghirardi, GC. and Marinatto, L. (2005). "Identical particles and entanglement." Optics and Spectroscopy, 99: 386-390.

Ghirardi, GC., Marinatto, L., and Weber, T. (2002). "Entanglement and properties of composite quantum systems: A conceptual and mathematical analysis." Journal of Statistical Physics, 108: 49-122.

Haag, R. (1993). Local Quantum Physics (Fields, Particles, Algebras). Berlin: Springer Verlag.

Harshman, N. L. (2012). "Observables and entanglement in the two-body system." AIP Conference Proceedings, 1508: 386-390.

Harshman, N. L. and Ranade, K. S. (2011). "Observables can be tailored to change the entanglement of any pure state." Physical Review A, 84: 012303.

Harshman, N. L. and Wickramasekara, S. (2007). "Galilean and dynamical invariance of entanglement in particle scattering." Physical Review Letters, 98: 080406.

Huggett, N. and Imbo, T. (2009). "Indistinguishability." Pp. 311-317 in D. Greenberger, K. Hentschel, and F. Weinert (eds.), Compendium of Quantum Physics. Berlin-New York: Springer.

Iguri, S. and Castagnino, M. (1999). "The formulation of quantum mechanics in terms of nuclear algebras." International Journal of Theoretical Physics, 38: 143-164.

Krause, D. (1992). “On a quasi-set theory.” Notre Dame Journal of Formal Logic, 33: 402-411.

Kuhlmann, M. (2010). The Ultimate Constituents of the Material World - In Search of an Ontology for Fundamental Physics. Frankfurt: Ontos-Verlag. 
Ladyman, J. (1998). "What is structural realism?" Studies in History and Philosophy of Science, 29: 409-424.

Ladyman, J., Linnebo, Ø., and Bigaj, T. (2013). "Entanglement and non-factorizability.” Studies in History and Philosophy of Modern Physics, 44: 215-221.

Lee, H.-W. and Kim, J. (2000). "Quantum teleportation and Bell's inequality using single-particle entanglement." Physical Review A, 63: 012305.

Lombardi, O. (2019). "The modal-Hamiltonian interpretation: Measurement, invariance, and ontology." Pp. 32-50 in O. Lombardi, S. Fortin, C. López, and F. Holik (eds.), Quantum Worlds: Perspectives on the Ontology of Quantum Mechanics. Cambridge: Cambridge University Press.

Lombardi, O. and Castagnino, M. (2008). "A modal-Hamiltonian interpretation of quantum mechanics." Studies in History and Philosophy of Modern Physics, 39: 380-443.

Lombardi. O. and Dieks, D. (2016). "Particles in a quantum ontology of properties.” Pp. 123-143 in T. Bigaj and C. Wüthrich (eds.), Metaphysics in Contemporary Physics. Leiden: BrillRodopi.

Messiah, A. M. (1961). Quantum Mechanics, Volumes I and II. North Holland: Amsterdam.

Messiah, A. M. L. and Greenberg, O. W. (1964). "Symmetrization postulate and its experimental foundation." Physical Review B, 136: 248-267.

Muller, F. A. and Leegwater, G. (2020). "The case against factorism: On the labels of $\otimes$ - factor Hilbert- spaces of similar particles in quantum mechanics." Journal for General Philosophy of Science, on line first: https://link.springer.com/article/10.1007/s10838-020-09514-6.

Myrvold, W. (2018). "Philosophical issues in quantum theory.” In E. N. Zalta (ed.), The Stanford Encyclopedia of Philosophy, (Fall 2018 Edition), URL = https://plato.stanford.edu/archives/fall2018/entries/qt-issues/ 
Myrvold, W., Genovese, M., and Shimony, A. (2020). "Bell's theorem.” In E. N. Zalta (ed.), The Stanford Encyclopedia of Philosophy, (Fall 2020 Edition), URL = https://plato.stanford.edu/archives/fall2020/entries/bell-theorem/.

O'Leary-Hawthorne, J. (1995). "The bundle theory of substance and the identity of indiscernibles." Analysis, 55: 191-196.

Pan, J.-W., Bouwmeester, D., Weinfurter, H., and Zeilinger, A. (1998). "Experimental entanglement swapping: Entangling photons that never interacted." Physical Review Letters, 80: 3891-3894.

Redhead, M. (1980). "Models in physics." The British Journal for the Philosophy of Science, 31: $145-163$.

Redhead, M. and Teller, P. (1992). "Particle labels and the theory of indistinguishable particles in quantum mechanics.” The British Journal for the Philosophy of Science, 43: 201-218.

Schlosshauer, M. (2007). Decoherence and the Quantum-to-Classical Transition. HeidelbergBerlin: Springer.

Scholz, V. B. and Werner, R. F. (2008). “Tsirelson’s problem.” arXiv:0812.4305.

Segal, I. E. (1947). "Irreducible representations of operator algebras." Bulletin of the American Mathematical Society, 53: 73-88.

Shi, Y. (2003). "Quantum entanglement of identical particles." Physical Review A, 67: 024301.

Tarski, A. (1941). "On the calculus of relations.” The Journal of Symbolic Logic, 6: 73-89.

Terra Cunha, M. O., Dunningham, J. A., and Vedral, V. (2007). "Entanglement in single-particle systems." Proceedings of the Royal Society A, 463: 2277-2286.

van Fraassen, B. C. (1985). "Statistical behaviour of indistinguishable particles: Problems of interpretation." Pp. 161-187 in P. Mittelstaedt and E. W. Stachow (eds.), Recent Developments in Quantum Logic. Mannheim: B.I. Wissenschaft.

van Fraassen, B. C. (1991). Quantum Mechanics: An Empiricist View. Oxford: Clarendon Press. 
Viola, L. and Barnum, H. (2010). "Entanglement and subsystems, entanglement beyond subsystems, and all that." Pp. 16-43 in A. Bokulich and G. Jaeger (eds). Philosophy of Quantum Information and Entanglement. Cambridge: Cambridge University Press.

Wilson, J. (2017). "Determinables and determinates." In E. N. Zalta (ed.), The Stanford Encyclopedia of Philosophy, (Spring 2017 Edition), URL = https://plato.stanford.edu/archives/spr2017/entries/determinate-determinables/.

Zanardi, P. (2001). "Virtual quantum systems.” Physical Review Letters, 87: 077901.

Zanardi, P., Lidar, D. A., and Lloyd, S. (2004). "Quantum tensor product structures are observable induced." Physical Review Letters, 92: 06042.

Żukowski, M., Zeilinger, A., Horne, M. A., and Ekert, A. K. (1993). "Event-ready-detectors. Bell experiment via entanglement swapping.” Physical Review Letters, 71: 4287-4290. 